\title{
New diagnostics, available care offer hope to patients with drug-resistant tuberculosis
}

I t's bad enough to live in a country with the world's highest prevalence of both HIV and tuberculosis (TB). But at least with TB, the treatment is relatively quick - just six months - and effective. Unless of course, you're burdened with drug resistant TB (DR-TB). Then it's a different story entirely: up to two years of highly toxic chemotherapy drugs, with horrible adverse effects including, in extreme cases, psychosis and deafness. And that's assuming you get a diagnosis in time.

Until a few years ago, diagnosis was a big problem in the Kingdom of Swaziland. First you had to access the standard TB test - in itself problematic for the predominately rural population, as the test was only offered in health centres and hospitals, necessitating a lot of travel and expense. If you did get there and dutifully coughed up your sputum, you then waited up to three months for results while your sample was taken to the National Tuberculosis Reference Laboratory in the capital city of Mbabne to be cultured. If the sputum smear was positive and you took the short-course chemotherapy drugs but they didn't work, then another culture would be taken, this time to be sent to a lab in South Africa, to test for DR-TB. Another three months.

"Patients could be dead by the time the diagnosis arrived," says Diana Gomez, clinical microbiologist for Médecins sans Frontières (MSF) in Swaziland.

But that was before. Now testing and treatment is available in many clinics, much closer to home for rural Swazis. MSF works in Shiselweni and Manzini, two of the country's four regions. In Shiselweni, TB testing and treatment used to be available in just three locations, but now it's in all 22 clinics, plus a special TB wing has opened at a health centre. In Manzini, separate test-

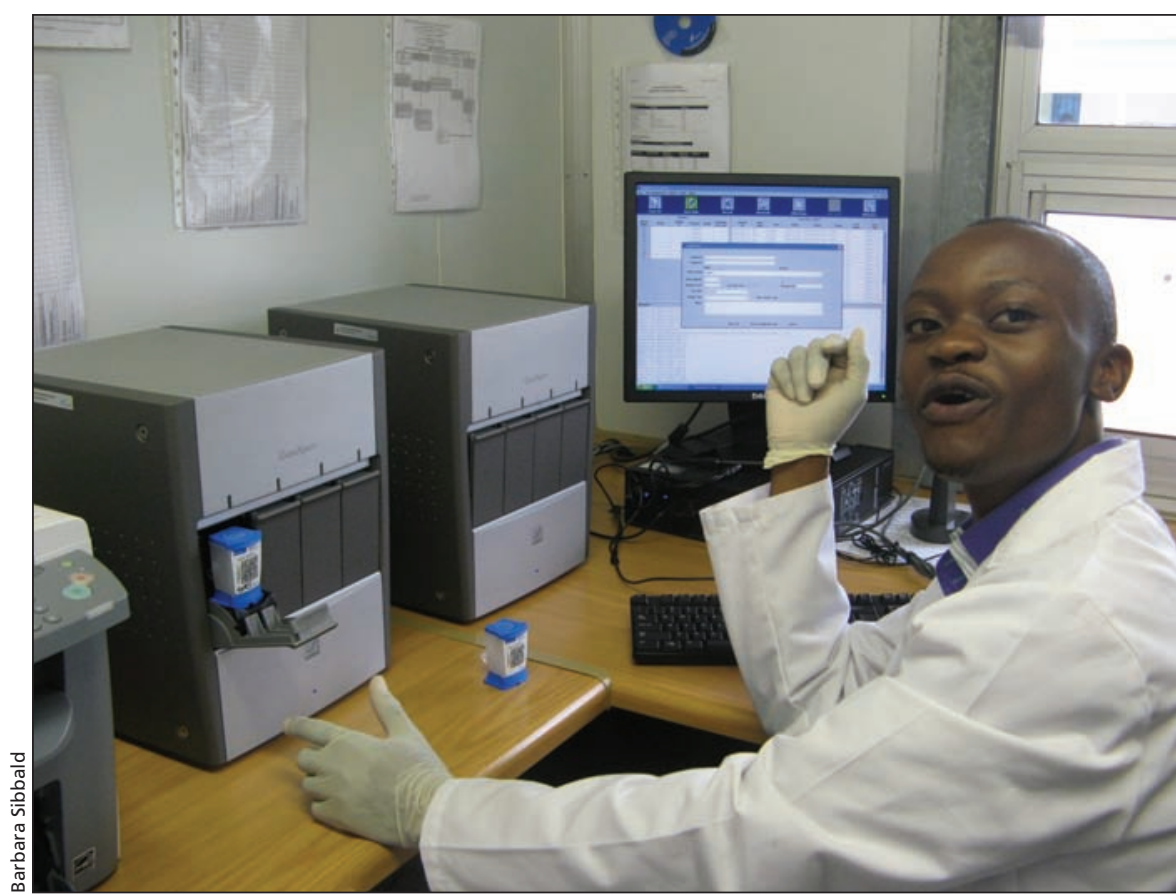

Tuberculosis testing is "way easier and very accurate" using a GeneXpert machine, says Mpumelelo Sifundza, a lab assistant at a Médecins sans Frontières clinic in Matsapha, Swaziland. "Before we prepared six slides."

ing and treatment facilities were constructed at a hospital and three clinics.

In addition, testing is now much faster. Beginning in 2011, MSF set up GeneXpert machines in three Swaziland facilities that detect $\mathrm{TB}$ and rifampicin resistance within two hours. The machine also detects $45 \%$ more cases and provides a personalized prescription.

A recent assessment of GeneXpert at 25 sites in 18 countries shows an overall $50 \%$ increase in diagnosis of TB compared to the sputum smear microscopy (http://uwclh.conference 2 web.com/content/1678/details?from _view=all\&view_address=events $\% 3 \mathrm{D} 3$ \%26groups\%3D1\%26sessions\%3D300).

Gomez says: "It has revolutionized detection."

Swaziland's TB incidence rate is 1275 cases per 100000 people. That means there are nearly 15000 people with TB. Neighbouring South Africa has 980 cases per 100000 and Mozambique has 750.
"The high prevalence is because of high HIV," says Dr. Kazi Arif Uddin, the physician in charge of DR-TB at the Matsapha MSF health clinic in the Manzini region.

In Swaziland, $80 \%$ of people with TB also have HIV, which is twofold higher than the next highest country, says Jonathan Gass, an epidemiologist with MSF who is based in Manzini. "It's a very rare prevalence; normally you see around 20\%."

A 2010 United States Centers for Disease Control and Prevention report assessing TB in Swaziland stated that the "findings assert the need for widescale intervention" (wwwnc.cdc.gov/eid /article/18/1/11-0850_article.htm). Yet, of the estimated 15000 patients, only about 11000 are enrolled in treatment. Alarmingly, $7.8 \%$ of new cases and $33 \%$ of retreatment cases are drug resistant. Of the approximately 1100 people with DR-TB, MSF was treating 179 in 
two regions, as of June 2012, while the ministry of health treats about 400 .

One of the barriers to getting people on treatment is the stigma associated with TB. "It's more than HIV," says Uddin, "because of its infectious nature. People hide it from their partners. ... They hide it from their employer because they may get fired."

DR-TB develops when patients fail to complete their full course of treatment; when health care providers prescribe the wrong treatment, or administer the wrong dose for the wrong period of time; when the supply of drugs is interrupted; or when the drugs are poor quality. DRTB is a particular concern for people with weakened immune systems, such as those living with HIV/AIDS.

It's possible to cure regular TB within six months, but DR-TB requires intensive treatment for up to two years with chemotherapy drugs that have toxic adverse effects, are less effective and are 50 to 200 times more expensive than regular TB treatment. The good news is that TB drugs are free (paid for through MSF or the government). The bad news is that the treatment for DRTB kicks off with six months of daily injections and many patients don't stick with the regime because of the adverse effects, including hearing impairment (which affects $15 \%$ of these patients) and psychosis (10\%); most who end up in hospital suffer from liver toxicity. Almost $70 \%$ experience at least one adverse effect and in 2010, 14\% died. In comparison, the mortality rate for Swazi patients with regular TB in 2011 was $8.5 \%$. According to MSF, TB is the leading cause of morbidity and mortality among Swazis living with HIV.

At Shiselweni's health centre, the mortality rate among multi-drug resistant TB patients is higher than the norm: about $16 \%$, says TB physician Dr. Munyaradzi Nyamutora. This is mostly due to coinfection and adherence problems. "They take 15-20 tablets two times a day." At least treatment support workers now give injections in the community, instead of patients travelling to clinics.

MSF also does intensive case finding, hosts support groups and engages treatment supporters, often neighbours, to watch patients take their drugs and provide counselling. It also provides patients with TB with about $\$ 87$ a month during the intensive phase of therapy, \$12.50 during continuation, plus a food package.

The more difficult cases continue to go to the Moneni TB hospital in Manzini. Uddin does weekly rounds at the hospital as one of five or so doctors who work there. "The quality of care at Moneni is very compromised," Uddin says. "We receive patients [at the MSF health centre] who have been there six months or a year, but badly managed in terms of regimen, doses and adherence. They toss their PAS [Para-aminosalicylic acid, one of the more toxic drugs in the cocktail] packets out the window. There's no DOTS [direct observed treatment short course]."

Money is not the problem, he adds. "If patients are not adhering, everything is not working. The problem is that the treatment is toxic. It makes them sick."

Despite all the advances, the problem of adherence isn't going away for patients with DR-TB. "The longer the treatment, the more likely you are to experience side-effects," says Uddin. "Two years is a long time." — Barbara Sibbald, CMAJ

CMAJ 2013. DOI:10.1503/cmaj.109-4341

Editor's note: Fourth of a multipart series. Barbara Sibbald's accommodation and transportation while in Swaziland were provided by Médecins sans Frontières.

Part I: Making sense of the world's highest HIV rate (www.cmaj.ca /lookup/doi/10.1503/cmaj.109-4315).

Part II: The face of an epidemic (www.cmaj.ca/lookup/doi/10.1503 /cmaj.109-4320).

Part III: Responding to Swaziland's dual epidemic (www.cmaj.ca/lookup /doi/10.1503/cmaj.109-4334).

Next: "I never gave up hope" 